\title{
PHOTODEGRADATION OF LOWER POLYBROMINATED DIPHENYL ETHER CONGENERS IN INDOOR AIR - MODEL STUDIES
}

\author{
Amelia Staszowska' \\ 1 Department of Indoor and Outdoor Air Quality, Faculty of Environmental Engineering, Lublin University of \\ Technology, Nadbystrzycka 40B, 20-618 Lublin, Poland, e-mail: a.staszowska@pollub.pl
}

Received: 2017.03.10 Accepted: 2017.04.04 Published: 2017.05 .02

\begin{abstract}
Polybrominated diphenyl ethers (PBDEs) are additive flame retardants which have found applications in polymers for many consumer plastic and electronic goods. Wide use of these chemicals has led to their extremely high concentrations in the indoor environments. This paper presents an exploratory study of the photodebromination of five individual lower polybrominated diphenyl ether congeners (BDE-28, BDE-47, BDE-99, BDE-100 and BDE-101) irradiated with white light and different UV (A, $\mathrm{B}, \mathrm{C})$ light ranges. The capability of photodegradation of lower PBDEs under room lights was proven by the debromination which occurred with various effectiveness for the selected PBDE congeners and depended of light source. Almost all PBDE congeners showed the greatest decay when exposed to UV-C, with the exception of BDE-100. The decay of irradiated congeners occurred rapidly during the first $5 \mathrm{~min}$ of experiment. The final degradation varied from 38\% decay (BDE-28, UV-C) to $94 \%$ (BDE-47, UV-C). These findings can help in predicting PBDE behavior in indoors and also be useful in the design of PBDE remediation processes.
\end{abstract}

Keywords: PBDEs, indoor air quality, flame retardants, photolytic degradation

\section{INTRODUCTION}

Nowadays, people in modern societies spend from $80 \%$ to $95 \%$ of their time in indoor environments [Schweizer et al., 2007]. According to the World Health Organization [2014], the indoor air quality has a greater impact on the health, wellbeing and productivity of occupants than the outdoor air pollution. Moreover, occupants of indoor environments are often exposed to chemicals that can be mostly attributed to indoor sources such as furnishing, upholstery, electronic devices, cleaning or cooking [Tham, 2016]. Newly constructed buildings must obey strict demands of energy loss and become more and more airtight, which leads to the reduction of natural infiltration and, in consequence, may cause an increase in contaminant levels. One of the most frequently detected group of indoor semivolatile organic pollutants are polybrominated diphenyl ethers (PBDEs). They are a class of additive brominated flame retar- dants (BFRs), extensively used in a wide range of polymer consumer products [Alaee and Wenning, 2002]. From the chemical point of view, PBDEs are synthetic aromatic compounds, diaryl ethers wherein one to ten hydrogen atoms have been replaced by atom/atoms of bromine in the positions from 2 to 6 , which finally gives 209 different congeners divided into ten groups of homologues (with an equal number of bromine substituents). PBDEs with one to five bromine atoms in a molecule are named lower or lighter BDEs, and those with six to ten bromines - higher BDEs, respectively [Alaee et al., 2003]. PBDEs were manufactured as three commercial mixtures called PentaBDE, Octa-BDE, and Deca-BDE. Congeners are not chemically bound to the polymer matrix but only physically mixed into it during manufacturing. The amount of PBDEs in the final product can reach 10 to $30 \%$ of weight loadings [EHC 162, 1994]. Because of the economic reasons and high flame retardancy properties, PBDEs have found 
application mostly in plastic used for electronic and electric appliances (computer casings, circuit boards, TV-sets), furnishing, upholstery, building materials, and even textiles [Allen et al., 2007].

The chemical and toxicological PBDE properties vary in respect to the degree of bromination. Lower PBDE congeners, especially with 2.2', 4.4' - bromine position, are the most toxic and characterized by the greatest bioaccumulation [Darnerud et al., 2007]. They can disrupt endocrine system, cause neurotoxic and cancerogenic health effects. In 2009, congeners which are components of Penta-BDE and Octa-BDE mixtures were added to the Annex A of the Stockholm Convention dedicated to Persistent Organic Pollutants. Although, the production of PBDEs has been ceased in the beginning of XXI century, significant volumes of these chemicals are still present in consumer products. International scientific data show that this class of BFRs became ubiquitous in wildlife and abiotic compartments of the environment all over the world, even in remote polar regions. The highest PBDE concentrations were found indoors [Butt et al., 2004; Harrad et al., 2006].

PBDEs may enter indoor air via a number of pathways, but the most important are evaporation (for lower BDE congeners) and abrasion (for heavy BDE congeners) of the goods during their service life. Many halogenated polycyclic organic compounds (polychloryniated biphenyls, polychlorinared and polybrominated dioxins and furans, perfluorinated compounds) are prone to undergo abiotic transformations in the environment [Bezarez-Cruz et al., 2004]. One of the possible pathways is the direct photolysis which leads to dehalogenation of irradiated chemical. PBDE phototransformation (mainly debromination) was confirmed in various abiotic media, predominantly in organic solvents [Barcellos da Rosa et al., 2003; Christiansson et al., 2009; Fang et al., 2008] and on mineral surfaces [Ahn et al., 2006; Kajiwara et al., 2008]. Peterman et al. [2003] reported on sunlight photolysis of a mixture of $39 \mathrm{PBDE}$ congeners in triolein. Reductive debromination of five individual PBDE congeners was examined by Sanchez-Prado et al. [2005] using solid-phase microextraction fibres. Granneli et al. [2011] reported reductive photodebromination of nonabrominated BDE congeners and analyzed the octabrominated products. Another study on photolysis of PBDEs was performed by Eriksson et al. [2004]. They measured photodegradation rate of 15 individual PBDE congeners in methanol/ water (8:2) solution, four congeners in tetrahydrofuran and nine congeners in methanol. Photodegradation of decabromodiphenyl ether in house dust under natural sunlight was described by Stapleton et al. [2008].

In indoor environments, PBDEs can be found in gas phase or sorbed on solid particles such as settled dust, particulate matter, and on impervious surfaces (windows, furniture, interior walls) [Cetin and Odobasi, 2011]. White light and UV light are intentionally applied by the user. Optical radiation is used for lighting. UV radiation has been found applications to improve indoor air quality in the process of air duct disinfection and to remove odors from the air.

The aim of the paper was to investigate the debromination of five individual lower PBDE congeners (BDE-28, BDE-47, BDE-99, BDE100 , BDE-101) under different light ranges (UVA, UV-B, UV-C and sun light). In the course of this study the photoproducts of debromination were identified, but results of these measurements are not included in this paper. The selected congeners are the main components of commercial mixtures Penta, and have the internal arrangement of the atoms of bromine in position 2.2", 4.4' which results in their high toxicity with respect to living organisms.

\section{MATERIALS AND METHODS}

\section{Chemicals}

All chemicals used for PBDE analysis were of high purity. Organic solvents (GC and HPLC grade) were purchased from T.J. Baker (POCH, Poland). PBDE analytical mixture standards series BDE-CVS-F containing $\mathrm{BDE} 28,47,85,99$, 100,101 congeners, as well as mass labeled $\left({ }^{13} \mathrm{C}\right)$ PBDE recovery solution BDE-MFX containing BDE 77L and 138L were obtained from Wellington Laboratories (Tusnovics, Poland). Additional standard solutions of native and mass labeled $\left({ }^{13} \mathrm{C}\right)$ PBDE individual BDE 28, 47, 85, 99, 100, 101 congeners were obtained from Wellington Laboratories and from AccuStandards (mixture M-527 BDE) (Tusnovics, Poland).

\section{Irradiation experiments}

The experimental set-up comprised a chamber with the outer and internal walls made from 
stainless steel. Between the walls, the active carbon filter was provided. The UV-lamp and white light lamp irradiation experiments were performed with lamps that can typically be employed in room lightening or indoor air cleaning devices. Lamps for exposures were placed on a movable grate. Three different UV irradiation light sources representing the wavelengths of UV-A, UV-B, and UV-C were used in this investigation. For UV-A range, $15 \mathrm{~W}$ linear fluorescent lamp was used (Philips, $365 \mathrm{~nm}$ ). The UV-B light source was medical lamp 01 PL-S, 15W/01 (Philips, $311 \mathrm{~nm}$ ) and UV-C irradiation tests were made with HNS bactericidal ( $254 \mathrm{~nm}, 15 \mathrm{~W})$ lamp (Philips). White light was simulated by MASTER TL-D Super 80, lamp (15W, Philips). During all irradiation experiments temperature and relative humidity were controlled and established to $22 \pm$ $0.2^{\circ} \mathrm{C}$ and $50 \pm 5 \%$, respectively.

Studies of the effect of UV-A, UV-B, UV-C and white light irradiation on PBDE debromination were started by the PBDE degradation model system which mimics the organic window film. The deposited PBDE individual congeners (in the amount of $2500 \mathrm{ng}$ ) were subjected to irradiation for $5,15,30,45$, and 60 minutes, respectively. At the same time, a similarly prepared reference sample (sample "0") - not exposed to irradiation - was kept in the dark, under the same conditions of temperature and relative humidity conditions as experiment ones). Three replicates for all individual irradiated PBDE congeners were made to check the reproducibility of each photodegradation experiment and to obtain more reliable results.

\section{Analytical procedure}

After irradiation, slides with PBDEs were rinsed with organic solvent mixture in dark room conditions. There was no need to perform any additional purification step. Next, the extract volume was reduced to $100 \mu \mathrm{L}$ under gentle nitrogen stream. Identification and quantification of irradiated individual PBDE congeners were performed by means of a Trace Ultra gas chromatograph (Thermo, USA) coupled with PolarisQ (Thermo, USA) mass detector (ion trap) operating in electron impact ionization $(70 \mathrm{eV})$ and selected ion monitoring mode (SIM). Chromatographic separation was conducted on $60 \mathrm{~m}$ RTx-5ms (Restek, USA) capillary column, $0.25 \mathrm{~mm}$ i.d.; $0.18 \mu \mathrm{m}$ film thickness. Helium 5N (Linde GAZ, Poland) was used as a carry gas (constant velocity $40 \mathrm{~cm} \cdot \mathrm{s}^{-}$ $\left.{ }^{1}\right)$. Oven temperature programming was as follow: from $85^{\circ} \mathrm{C}(0.5 \mathrm{~min}$ hold $)$ with ramp $15^{\circ} \mathrm{C} \cdot \mathrm{min}^{-1}$ to $210^{\circ} \mathrm{C}$, then ramp $5^{\circ} \mathrm{C} \cdot \mathrm{min}^{-1}$ to $320^{\circ} \mathrm{C}(20 \mathrm{~min}$ hold). Injector (PTV, splitless mode) temperature was set at $320^{\circ} \mathrm{C}$. Transfer line, ion source temperature were kept constant at $275^{\circ} \mathrm{C}$ and $250^{\circ} \mathrm{C}$, respectively. A total of 5 (tri- to pentaBDE) congeners (BDE-28, BDE-47, BDE-99, BDE-100 and BDE-101) were measured in extracts and quantified using the internal standard method.

The identification of photoirradiated PBDE congeners and their photoproducts was based on the comparison of their mass spectra and retention times with reference native and ${ }^{13} \mathrm{C}$-labbeled PBDE standards. The resulting mass spectra were analyzed using the Xcalibur software and databases of mass spectra from NIST.05 library and Wiley $8^{\text {th }}$ edition.

GC detection limits were estimated at 0.5 $n g \cdot L^{-1}(S / N \geq 3$ ) and the extract (from 17 to BDE-BDE-153). The limit of quantification was determined to be 10 pg per dosing $(\mathrm{S} / \mathrm{N} \geq 5)$. Linearity of the method was better than of 10-500 to all congeners except BDE-153 (better than 10-200). The relative standard deviation (RSD) of the 5 samples analysis was less than $16 \%$ for the congeners from BDE-17 to BDE-101. In model studies at concentrations above $500 \mathrm{ng} \cdot \mathrm{g}-$ ${ }^{1}$, RSD was less than $10 \%$ in the assays GC/MS for analyzed congeners.

\section{Quality assurance and quality control}

Taking into account that PBDEs can undergo of phototransformation under light, a special care was given to prevent any possibilities of artificial or natural light exposure during their storage and analysis. All glassware used during the experiments was pre-washed with mixture of organic solutions (GC and HPLC grade), rinsed with water and redistilled water (MiliQ), baked at $450{ }^{\circ} \mathrm{C}$ and finally wrapped in aluminum foil to protect from possible secondary contamination.

The nomenclature for PBDE congeners used in this study is in analogy to the polychlorinated biphenyl numbering system established by Ballschmiter and Zell in 1980.

\section{RESULTS}

During the irradiation of model systems, individual PBDE congeners deposited on the surface 
of a glass slide, changes in the irradiated mass congeners were observed, which was the evidence of degradation.

Dark control experiments (called sample " 0 “) for each congener showed no disappearance of the congener or appearance of photodebromination products. In addition, no potential debromination was observed in the starting ( $0 \mathrm{~min})$ film systems.

Figures 1 to 5 report changes in mass of individual PBDE congeners during irradiation with different light sources.

\section{BDE-28 (2,4,4'-tribromodiphenyl ether)}

After the first 5 minutes of exposure, a slight weight loss of BDE-28 was observed only for the samples irradiated UV-C, it amounted to 6\%. After 15 minutes of exposure, degradation BDE-28 for all ranges of irradiation was confirmed. It was the greatest for a range of UV-C (15\%), while for the other UV ranges, it approximated $6 \%$. After 30 minutes of exposure to UV-C, the degradation reached $20 \%$, whereas for the UV-A, UV-B and white light, it amounted only to $9 \%, 12 \%$ and
$16 \%$, respectively. In 45 minutes of exposure with UV-C, the degradation was assumed as $28 \%$, and $20 \%$ for UV-B and white light, and $12 \%$ for UVA. After 60 minutes of the experiment, the degradation of BDE-28 reached $38 \%$ for UV-C, $25 \%$ for UV-B and white light, and $20 \%$ for UV-A.

The obtained results confirm that the BDE28 containing 3 bromine atoms in molecule underwent photodegradation and the most effective range of radiation was UV-C. Similar results of BDE-28 debromination were found for UV-B and white light. The smallest impact on the debromination of BDE-28 showed a UV-A range.

\section{BDE-47 (2,2',4,4'-tetrabromodiphenyl ether)}

The decay of BDE-47 during irradiation occurred more rapidly than in the case of BDE-28 (Figure 2).

After the first 5 minutes of exposure, BDE47 debromination for all types of radiation was observed. The biggest change in the mass of the treated congener, amounting to $87 \%$, was determined for the UV-C radiation. Then BDE-47

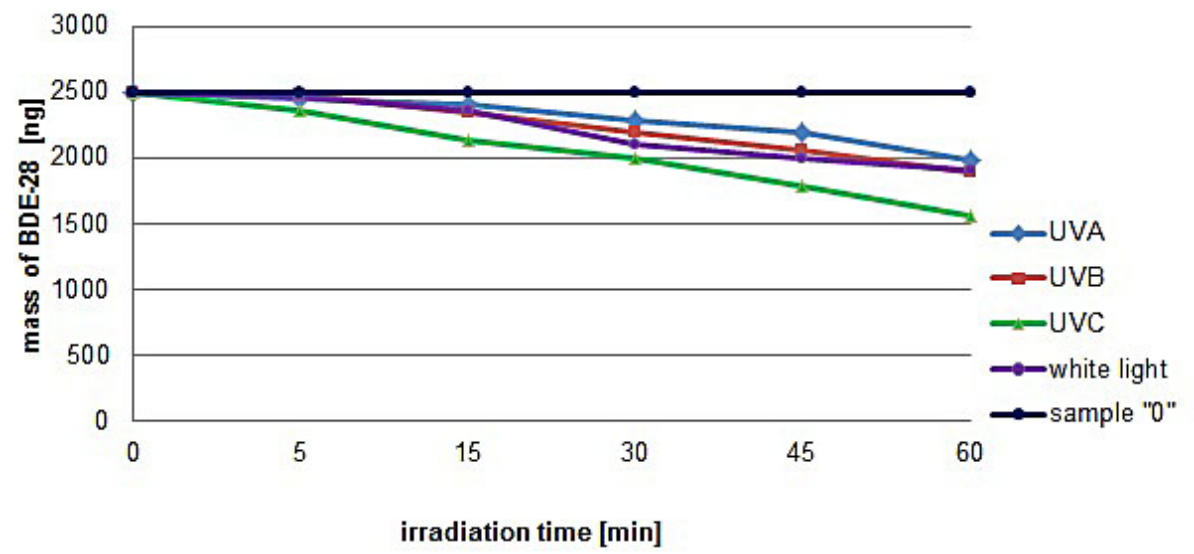

Figure 1. The influence of optical radiation on the degradation of BDE-28

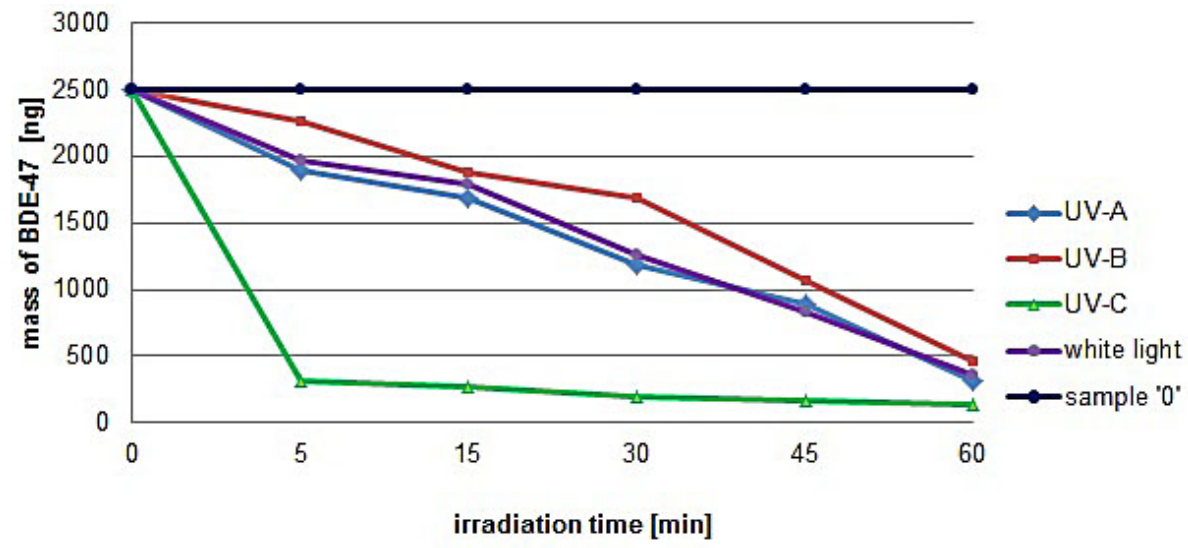

Figure 2. The influence of optical radiation on the degradation of BDE-47 
mass was decreasing subsequently and finally reached $94 \%$ decay in 60 min of experiment. The observed BDE-47 degradation under UV$\mathrm{B}, \mathrm{UV}-\mathrm{C}$ and white light was not as rapid as for UV-A. The influence of UV-A and white light was similar. After 15 minutes of exposure, BDE47 degradation of initial mass was calculated as $33 \%$ for UV-A, $25 \%$ for UV-B, $89 \%$ for UV-C and $28 \%$ for white light, respectively. After 30 minutes the experiment, UV-B achieved 33\% degradation, UV-A $-52 \%$, UV-C $-92 \%$ and white light $-50 \%$. An increase in the degradation of BDE-47 was observed in 45 minutes, which reached $64 \%$ (UV-A) and, similarly, 67\% for white light. For UV-C, the irradiated congener weight loss was calculated as $93 \%$. The lowest degradation was observed after 45 minutes for the UV-B - only $57 \%$. After 60 minutes of the experiment, the weight loss of the treated congener was similar for all analyzed ranges of radiation. For UV-A it amounted to $87 \%$, for UV-B - 94\%, for UV-C $-94 \%$, and white light $-86 \%$. Thus, BDE47 , just as the BDE-28, degrade the most rapidly under the influence of UV-C, but after 60 minutes of exposure degradation, BDE-47 for UV-B and UV-C has reached the same value as for the UV-A and white light.

\section{BDE-99 $(2,2,4,4,5$-pentabromodiphenyl ether)}

Similarly to the BDE28 and BDE-47, the irradiation of UV-C range was most effective in the initial period (Figure 3).

After 5 minutes of the experiment, the UV-C caused a $62 \%$ decay of BDE-99, while the UV-A was only $27 \%$, UV-B - $19 \%$, and white light $28 \%$. After next 15, 30 and 45 minutes, a con- tinuing degradation of BDE-99 was observed; after 45 minutes it amounted $82 \%$ for UV-C, $71 \%$ for UV-B radiation, $76 \%$ for UV-A and $78 \%$ for white light. After 60 minutes, the final degradation of BDE-99 reached over $80 \%$ for all light sources. In summary, the BDE-99 degrades quickly under the influence of UV-C. The biggest change in the weight of the treated congener was observed after the first 5 minutes of the experiment. Then the process of degradation congener occurred much milder. A similar pattern of degradation of BDE-99 could be observed for the UV-A and white light.

\section{BDE-100 (2,2',4,4',6-pentabromodiphenyl ether)}

The congeners BDE-100 and BDE-101 are homologues of BDE-99. For any of them, no such rapid degradation occurred in the first minutes of the experiment, as in the case of BDE-99 (Figure 4).

After the first five minutes of the experiment, the largest degradation BDE-100 was obtained for UV-A - 24\%. A similar value was recorded also for UV-B $-22 \%$ and white light $20 \%$. After 15 minutes the degradation of BDE- 100 took place for the UV-B and UV-A, reaching 38\% and $39 \%$, respectively, whereas in the case of white light $-34 \%$. After 30 minutes, the decay of BDE100 initial mass under UV was detected. After 45 minutes of exposure, the UV-A degradation was the highest, but similar to the UV-B and white light. The lowest degradation of BDE-100 after 45 minutes was observed for UV-C radiation, although it has reached $57 \%$. After 60 minutes of exposure, the weight loss of BDE-100 was similar to the UV-A and UV-B and stood at $78 \%$ and

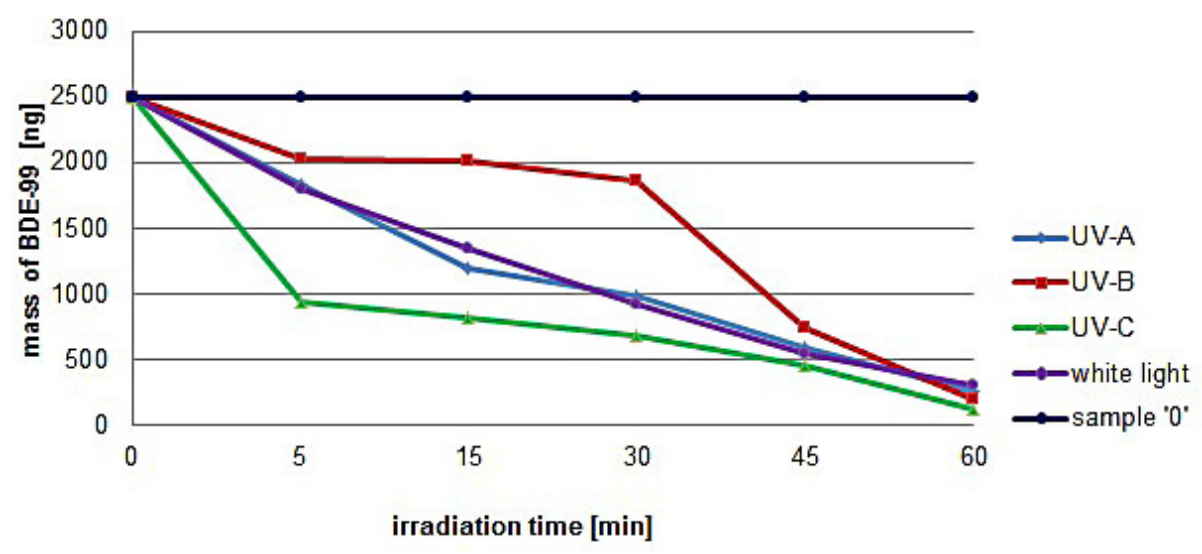

Figure 3. The influence of optical radiation on the degradation of BDE-99 


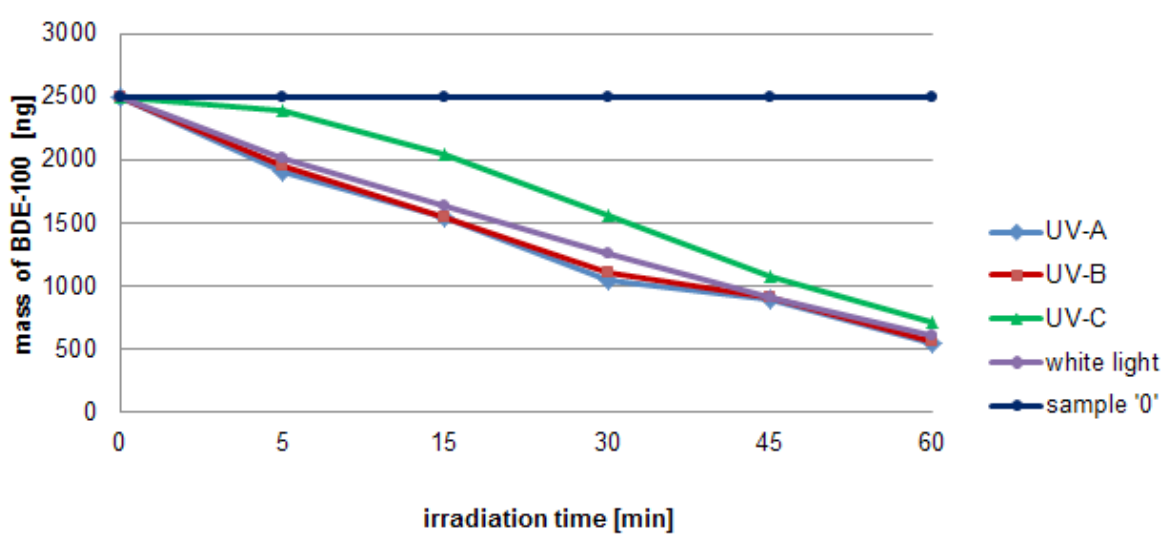

Figure 4. The influence of optical radiation on the degradation of BDE-100

$77 \%$, respectively. The differences between the UV-C light and white or longer were not as large as for shorter exposure times and amounted to $72 \%$ for UV-C and $76 \%$ for white light.

In summary, the initial degradation of BDE100 was the highest for UV-A and UV-B, but as the exposure time of BDE-100 congener increased, the differences in the degradation of BDE-100 under the influence of various ranges of optical radiation decreased. As a result, after 60 minutes of exposure, BDE-100 has degraded in more than $70 \%$ for all ranges of radiation.

\section{BDE-101 (2,2',4,5,5'- pentabromodiphenyl ether)}

In the first 5 minutes of experiment, the degradation of BDE-101 was reported only for UV-B (16\%). After another 15 minutes, BDE-101 started to decay for all ranges of radiation and was at a level of $6 \%$ for UV-A, $26 \%$ for UV-B , $12 \%$ for UV-C, and $11 \%$ for white light. At the half of experiment, the largest degradation of BDE-101 was detected for UV-B, reaching $40 \%$. For the other ranges, it was approximately $20 \%$ lower. After next 15 minutes, BDE-100 decayed at $60 \%$ for UV-B, 34\% for UV-A, 25\% for UV-C and 32\% for white light, respectively. At the end of irradiation, the largest degradation of BDE-101 was achieved by UV-B radiation $-78 \%$. In contrast, for the UV-A it amounted to $68 \%$, for the UV-C $33 \%$ and for white light $-65 \%$. Thus, in the case of BDE-101, the greatest decay effect was observed for UV-B and the least for UV-C which is different from the BDE-99 and BDE-100 results.

\section{CONCLUSIONS}

This study indicates that lower PBDE congeners can undergo room-light-induced debromination in indoor air. The results showed that (1) the efficiency of photodegradation varied and depended on amount of bromine atoms in congener and light source (2) the photodegradation of highly bromo-substitued PBDEs was faster than with smaller amount of bromines (3) the photodegradation conversion of BDE-28, BDE-47, BDE-99,

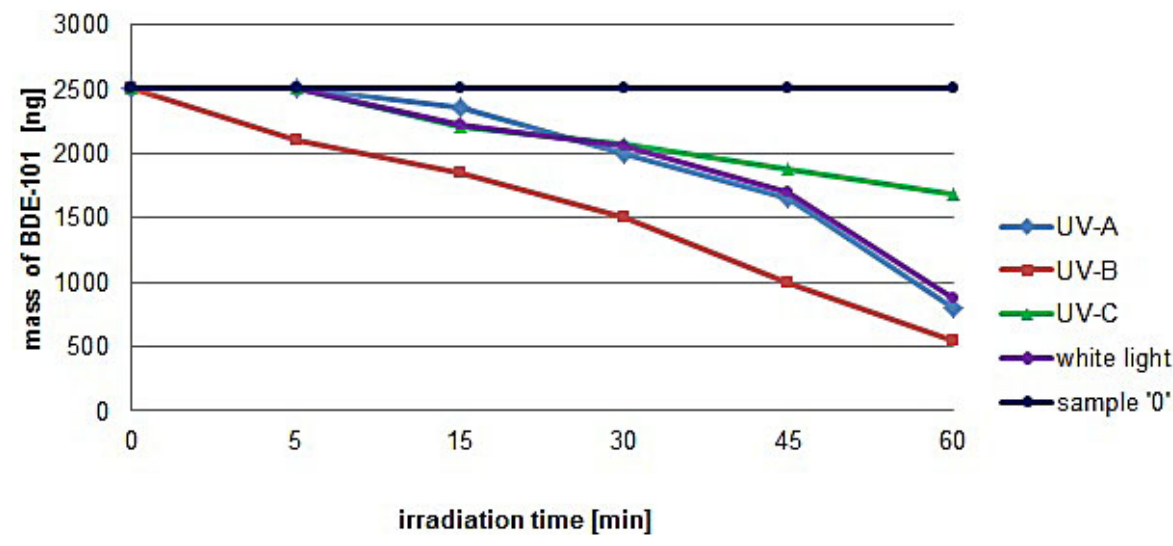

Figure 5. The influence of optical radiation on the degradation of BDE-101 
and BDE-101 was mostly affected by UV-C (4) the slowest degradation of lower PBDE congeners was observed for white light and UV-B ranges (for most of BDEs) (5) white light and UV-A showed similar pattern of effectiveness in degradation for analyzed PBDEs. The understanding of indoor air chemistry of lower PBDEs can be valuable for the exposure evaluation and searching for new effective remediation methods.

\section{REFERENCES}

1. Ahn M.Y., Filey T.R., Javert C.T., Nies L., Hua I., Bezares-Cruz J., 2006. Photodegradation of decabromodiphenyl ether adsorbed onto clay minerals, metal oxides, and sediments. Environmental Science \& Technology 40, 215 - 220.

2. Alaee M., Wenning R.J., 2002. The significance of brominated flame retardants in the environment: current understanding, issues and challenges. Chemosphere 46, 579-582.

3. Alaee M., Arias P., Sjödin A., Bergman A., 2003. An overview of commercially used brominated flame retardants, their application, their use patterns in different countries/ regions and possible modes of release. Environment International 29, 683-689.

4. Allen J., McClean M., Stapleton H., Nelson J., Webster T., 2007. Personal exposure to polybrominated diphenyl ethers (PBDEs) in residential indoor air. Environment Science \& Technology 41 , 4574-4579.

5. Ballschmiter K., Zell M., 1980. Analysis of polychlorinated biphenyls (PCB) by glass capillary gas chromatography. Fresenius Journal of Analytical Chemistry 302, 20-31.

6. Barcellos da Rosa M., Krüger H.U., Thomas S., Zetzsch C., 2003. Photolytic debromination and degradation of decabromodiphenyl ether, and exploratory kinetic study in toluene. Fresenius Environmental Bulletin 12, 940 - 945.

7. Bezares-Cruz J., Jafvert C.T., Hua I., 2004. Solar photodecomposition of decabromodiphenyl ether: products and quantum yield. Environmental Science \& Technology 38, 4149 - 4156.

8. Butt C.M., Diamond M.L., Ikonomou T.J., 2004. Spatial distribution of polybrominated diphenyl ethers in Southern Ontario as measured in indoor and outdoor window organic films. Environmental Science \& Technology 38, 724-731

9. Cetin B., Odobasi M., 2011. Polybrominated diphenyl ethers (PBDEs) in indoor and outdoor window organic films in Izmir, Turkey. Journal of Hazardous Materials 185, 784-791.

10. Christiansson A., Eriksson J., Techiel D., Bergman A., 2009. Identification and quantification of products formed via photolysis of decabromodiphenyl ether. Environmental Science and Pollution. Research. 16, 312-321.

11. Darnerud P.O., Eriksen G.S., Johannesson T., Larsen P., Viluksela M., 2001. Polybrominated Diphenyl Ethers: occurrence, dietary exposure, and toxicology. Environmental Health Perspectives 109, 49-68.

12. Environmental Heath Criteria 162: Brominated Diphenyl Ethers, Word Heath Organization, Geneva, 1994.

13. Eriksson J., Green N., Marsh G., Bergman Å., 2004. Photochemical decomposition of 15 polybrominated diphenyl ether congeners in methanol/water. Environmental Science \& Technology 38, 3119-3125.

14. Fang L., Huang J., Yu G., Wang L., 2008. Photochemical degradation of six polybrominated diphenyl ether congeners under ultraviolet irradiation in hexane. Chemosphere 71, $258-267$.

15. Granelli L., Eriksson J., Athanasiadou M., Bergman A., 2011. Reductive debromination of nonbrominated diphenyl ethers by sodium borohydride and identification of octabrominated diphenyl ether products. Chemosphere 82, 839-846.

16. Harrad S., Hazrati S., Ibarra C., 2006. Concentrations of polychlorinated biphenyls in indoor air and polybrominated diphenyl ethers in indoor air and dust in Birmingham, United Kingdom: implications for human exposure. Environmental Science \& Technology 40, 4633-4638.

17. Kajiwara N., Noma Y., Takigami H., 2008. Photolysis studies of technical decabromodiphenyl ether (DecaBDE) and ethane (DeBDethane) in plastics under natural sunlight. Environmental Science \& Technology 42, 4404-4409.

18. Peterman P.H., Orazio C.E., Feltz K.P., 2003. Sunlight photolysis of 39 mono-hepta PBDE congeners in lipid. Organohalogen Compounds 63, 357-360.

19. Sánchez-Prado L., González-Barreiro C., Lores M., Llompart M., Garcia-Jares C., Cela R., 2005. Photochemical studies of polybrominated diphenyl ethers (PBDEs) technical mixture by solid phase microextraction (SPME). Chemosphere 60, 922-928.

20. Schweizer C., Edwards R.D., Bayer-Oglesby 1., Gauderman W.J., Ilacqua V., Jantunen M.J., Lai H.K., 2007. Indoor time-microenvironment-activity patterns in seven regions of Europe. Journal of Exposure Science and Environmental Epidemiology $17,170-181$.

21. Stapleton H.M., Dodder N. G., 2008. Photodegradation of decabromodiphenyl ether in house dust by natural sunlight. Environmental Toxicology and Chemistry 27, 306-312.

22. Tham K.W., 2016. Indoor air quality and its effects on humans - a review of challenges and developments in the last 30 years. Energy and Buildings 130, 637-650.

23. World Health Organization, WHO 2014. 